\title{
Avaliação da capacidade funcional em idosos diabéticos
}

\author{
Functional capacity assessment in aged diabetic patients \\ Daniela Maria da Cruz dos Anjos ${ }^{1,2}$, Isabella Lima Araújo33, Vivian Morais Barros³, \\ Danielle Aparecida Gomes Pereira ${ }^{4}$, Daniele Sirineu Pereira ${ }^{1}$
}

\begin{abstract}
RESUMO I O diabetes mellitus (DM) tem associação com várias comorbidades que comprometem ainda mais a funcionalidade do idoso. Essa doença crônica está vinculada ao risco de incapacidade para realizar atividades rotineiras. O objetivo deste estudo foi comparar a capacidade funcional de idosos diabéticos e não diabéticos. Trata-se de uma pesquisa observacional transversal que avaliou 40 idosos, divididos em dois grupos, sendo 20 dia-

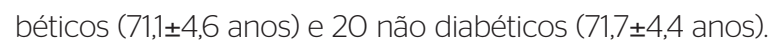
Os grupos foram semelhantes quanto ao gênero, idade e índice de massa corpórea (IMC). Para avaliação da capacidade funcional foram realizados o teste de desempenho físico modificado (TDMF) e o teste de caminhada de seis minutos (TC6). A percepção subjetiva do esforço foi avaliada por meio da escala de Borg modificada e o teste de sensibilidade pela percepção de um conjunto de seis monofilamentos de náilon de Semmes-Weinstein. Os dados foram analisados por meio do Mann-Whitney test. 0 grupo de idosos diabéticos percorreu menor distância no TC6 (396 $\pm 55,6 \times 481,5 \pm 73,1$ m; p=0,0003) e apresentou pior teste de sensibilidade quando comparado com os não diabéticos, mas nenhuma diferença foi encontrada no TDMF. Os idosos diabéticos relataram pior aptidão física quando comparados com os não diabéticos.
\end{abstract}

Descritores I diabetes mellitus; idoso; avaliação; envelhecimento.
ABSTRACT I Diabetes mellitus (DM) is associated with much comorbidity which compromise even more the aged functional capacity. This chronic illness is associated with the risk of disability to perform activities of daily living. The purpose of this study was to compare the functional capacity of diabetic and non-diabetic aged. It is a cross sectional study, that evaluated forty aged, dived in 2 groups, 20 diabetic ( $71.1 \pm 4.6$ years) and 20 non-diabetic ( $71.7 \pm 4.4$ years). The groups where similar in gender, age and BMI. To assess functional capacity, the Physical Performance Test Modified (PPTM) and the six minute walk test were performed. The rate of perceived exertion was examined through the Borg scale and the sensibility test through the perception of a group of six nylon Semmes-Weinstein monofilaments. Data were analyzed through Mann-Whitney test. The group of diabetic aged walked a shorter distance in the 6 minute walk test (396 $\pm 55.6 \times 481.5 \pm 73.1 \mathrm{~m} ; \mathrm{p}=0.0003)$ and showed worst sensibility test when compared to the non-diabetic elderIy, but no difference was found in the PPTM. The diabetic elderly showed inferior physical ability compared to the non-diabetic elderly.

Keywords I diabetes mellitus; aged; evaluation; aging.

\footnotetext{
Estudo desenvolvido no Centro Universitário de Belo Horizonte (UNIBH) - Belo Horizonte (MG), Brasil. 'Doutoranda no Programa de Pós-graduação em Ciências da Reabilitação da Escola de Educação Física, Fisioterapia e Terapia Ocupacional da Universidade Federal de Minas Gerais (EEFFTO/UFMG) - Belo Horizonte (MG), Brasil. ${ }^{2}$ Professora no Curso de Fisioterapia da Faculdade Estácio de Sá - Belo Horizonte (MG), Brasil.

${ }^{3}$ Fisioterapeuta graduada pela UNIBH - Belo Horizonte (MG), Brasil.

${ }^{4}$ Professora Doutora Adjunto do Departamento de Fisioterapia da EEFFTO/UFMG - Belo Horizonte (MG), Brasil.
}

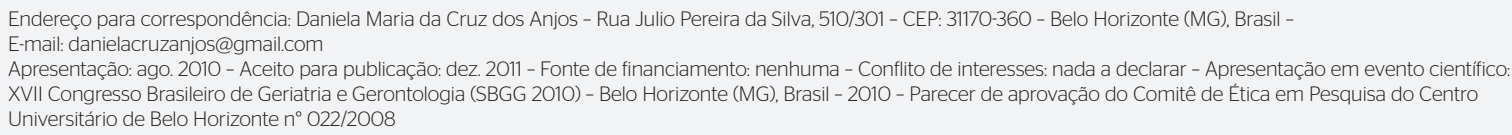




\section{INTRODUÇÃO}

O envelhecimento é um processo dinâmico no qual ocorrem alterações progressivas no organismo tornando-o mais susceptível a agressões intrínsecas e extrínsecas, aumentando de forma exponencial o aparecimento de doenças crônicas, como o diabetes mellitus (DM) ${ }^{1}$.

Uma das síndromes geriátricas mais graves é a incapacidade funcional. Os estudos transversais demonstram que o diabetes está associado a um duplo aumento no risco de incapacidade para realizar atividades de vida diária quando comparados indivíduos com e sem a doença ${ }^{2,3}$.

O DM tem associação com várias comorbidades que comprometem ainda mais a funcionalidade do idoso ${ }^{4}$. Com a diminuição da sensibilidade plantar e das informações provenientes dos mecanorreceptores, há também restrição sobre o equilíbrio em idosos diabéticos ${ }^{5,6}$. As quedas são mais prováveis de ocorrer durante atividades de locomoção, como caminhar, subir e descer escadas e desviar de obstáculos ${ }^{7}$. Nesses momentos, é fundamental a transferência de peso de um pé para o outro ${ }^{8}$, breves períodos de manutenção da postura sobre os membros inferiores e preservação da sensibilidade para realização das atividades de vida diária (AVD).

Gregg et al. ${ }^{2}$ demonstraram em seu estudo que, entre 6.588 indivíduos com 60 anos ou mais, 32\% das mulheres e $15 \%$ dos homens com DM relataram pelo menos uma incapacidade, como andar $400 \mathrm{~m}$, fazer tarefas domésticas ou subir escadas. Em comparação, 14\% das mulheres e $8 \%$ dos homens sem diabetes fizeram relatos parecidos. Frente a essas evidências de incapacidades e pior desempenho ao realizar tarefas do dia a dia, torna-se importante comparar o desempenho físico de idosos diabéticos com o de não diabéticos, por meio de testes que avaliem múltiplos domínios da função física e possam ser um indicador geral dele e também da mobilidade.

O conhecimento dessas possíveis diferenças pode orientar a atenção à saúde do idoso, visando à tomada de decisões e o planejamento de ações de educação, prevenção e promoção de saúde. Além disso, a limitação na capacidade funcional pode diminuir o convívio social e interferir no senso de bem-estar. Preservar a capacidade funcional é um dos principais objetivos da fisioterapia. Dessa forma, o objetivo desse estudo foi comparar a capacidade funcional de idosos diabéticos e não diabéticos.

\section{MATERIAIS E MÉTODOS}

Trata-se de uma pesquisa observacional transversal, que avaliou idosos com e sem DM, residentes na comunidade de Belo Horizonte e região metropolitana, sem distinção de raça e/ou condição socioeconômica, com idade igual ou superior a 60 anos. Este estudo foi aprovado pelo Comitê de Ética em Pesquisa do Centro Universitário de Belo Horizonte (UNIBH), em Minas Gerais, Brasil, sob o no 022/2008. Todas as voluntárias assinaram o termo de consentimento livre e esclarecido.

Participaram do estudo 40 idosos comunitários, divididas em dois grupos, com (G2) e sem (G1) DM. A presença da doença foi determinada por autorrelato, em resposta à pergunta: "O seu médico já lhe disse que você tem diabetes?”. Para participar do grupo de diabéticos, além de resposta afirmativa, ela deveria atender a pelo menos uma das seguintes condições: (1) glicemia de jejum $\geq 126 \mathrm{mg} / \mathrm{dL}$ ou (2) glicemia 2 horas após $75 \mathrm{~g}$ de glicose oral $\geq 200 \mathrm{mg} / \mathrm{dL}$ ou (3) uso de medicamento para controle do diabetes. Os exames laboratoriais foram realizados com até seis meses antes da coleta.

As participantes foram selecionadas por conveniência e recrutadas em Belo Horizonte e na região metropolitana. Os critérios de inclusão foram: homens ou mulheres com idade igual ou superior a 60 anos, residir na comunidade e ter marcha independente. Foram critérios de exclusão: alterações cognitivas detectáveis pelo miniexame do estado mental (MEEM), considerando os pontos de corte de acordo com a escolaridade ${ }^{9}$, presença de doenças neurológicas, dispositivos de auxílio à marcha, amputação de membros inferiores, doenças ortopédicas ou reumáticas que afetassem a marcha e presença de úlceras plantares ativas. Também foram excluídos os pacientes que estavam em tratamento fisioterápico e/ou que realizavam exercício físico de forma regular três vezes por semana ou mais ou com duração $\geq 150 \mathrm{~min} / \mathrm{sem}$.

Para a avaliação da capacidade funcional, foram realizados o teste de desempenho físico modificado (TDMF) e o teste de caminhada de seis minutos (TC6).

O TDMF é uma adaptação para a população brasileira do Physical Performance Test Modified ${ }^{10}$. Trata-se de um teste que avalia múltiplos domínios de função física, pela realização de tarefas que simulam atividades básicas e instrumentais de vida diária, em níveis diferentes e progressivos de dificuldade. É composto por sete itens funcionais, que correlacionam graus de incapacidade, perda de independência e mortalidade precoce, e por dois itens que se correlacionam com institucionalização e perda de independência. 
Ele é composto pelas tarefas: (1) pegar um livro e colocá-lo sobre a mesa; $(2)$ vestir e despir uma jaqueta; (3) pegar uma caneta no chão; (4) sentar e levantar de uma cadeira; (5) girar 360; (6) caminhar $15 \mathrm{~m}$; (7) subir um lance de escadas (10 degraus); (8) subir e descer um lance de escadas; (9) teste de Romberg progressivo. Cada item do TDMF apresenta diferentes níveis de desempenho baseados no tempo necessário para executar a tarefa. Apresenta um escore de 0 a 4 pontos em cada item, totalizando máximo de 36 pontos. Altos escores representam melhor desempenho. Foi analisada a proporção de indivíduos do grupo de DM e não DM que realizava a atividade do TDMF em um determinado tempo.

Já o TC6 é um teste de esforço submáximo que permite avaliação objetiva da condição física do indivíduo. Foram realizados dois testes, sendo o primeiro para aprendizado e, após $30 \mathrm{~min}$, o segundo.

Para a sua realização, o idoso foi orientado a percorrer a maior distância possível durante seis minutos, em velocidade autosselecionada, em um corredor de $30 \mathrm{~m}$. Foi preconizado o uso da frase: "você está indo bem", para a sua motivação, a cada minuto. A monitorização da frequência cardíaca e pressão arterial era obrigatoriamente avaliada antes do início do teste e após o término.

A percepção subjetiva de esforço foi avaliada por meio da escala de Borg modificada, antes do início do teste e ao final de seis minutos. Válida e confiável, ela consiste em uma escala vertical graduada de zero a dez unidades arbitrárias com expressões verbais correspondentes a um aumento progressivo do nível de percepção do esforço, em que zero representa nenhum sintoma e dez, sintoma máximo ${ }^{11}$. As variáveis utilizadas para a análise foram percepção subjetiva do esforço e distância percorrida.

O teste de sensibilidade foi realizado utilizando-se um conjunto de seis monofilamentos de náilon de Semmes-Weinstein. A percepção de cada monofilamento está associada a um gradiente de alterações sensitivas. $\mathrm{O}$ teste foi aplicado em nove territórios específicos do nervo tibial posterior, um do nervo fibular e um do nervo sural. Após definir o resultado de cada área do pé, o registro foi enquadrado em uma das cinco graduações ou estágios (1: sensibilidade normal, 2: sensibilidade protetora diminuída, 3: perda de sensibilidade protetora, 4: sensação profunda presente, 5: perda da sensação profunda $)^{12}$.

Para todos os participantes, diabéticos ou não, foram aplicados os mesmos testes.

\section{Análise estatística}

A análise estatística descritiva foi usada para todas as variáveis clínicas e demográficas do estudo. Aplicou-se o teste de Shapiro-Wilk para verificar se os dados apresentavam distribuição normal. Para as variáveis que demonstraram distribuição normal, usou-se o teste $t$ de Student, independente e para as que não apresentaram utilizou-se teste não paramétrico - teste de Mann-Whitney.

Para a comparação das variáveis, distância percorrida em metros, ICQ e escore final do TDMF, entre os grupos de idosos, foi realizado o teste de Mann-Whitney.

O teste $t$ de Student independente teve o propósito de verificar se existe diferença entre as médias das variáveis idade, peso, altura, índice de massas corpórea (IMC) e Borg final entre os grupos. Para comparar a proporção do gênero masculino do grupo $\mathrm{G} 1$ com a proporção do gênero masculino do grupo $G 2$, realizou-se um teste $Z$ para duas proporções. Também houve esta mesma análise para as variáveis do TDMF - pegar um livro, vestir e despir um casaco, pegar uma moeda no chão, levantar-se da cadeira, caminhar $15 \mathrm{~m}$, subir um lances de escada, subir quatro lances de escadas, girar $360^{\circ}$, romberg progressivo - e para o escore total e sensibilidade.

Em todos os testes estatísticos utilizados, foi considerado nível de significância $\alpha$ de $5 \%$. As análises foram realizadas no software estatístico Minitab, versão 15, 2007.

\section{RESULTADOS}

Foram avaliados 40 idosos, sendo 20 sem DM no G1 e 20 com DM no G2. As características demográficas e clínicas são apresentadas na Tabela 1 . Os grupos foram semelhantes quanto ao gênero, idade e IMC; porém, foi encontrada diferença estatisticamente significativa entre os grupos em relação ao índice cintura quadril (ICQ).

No teste de sensibilidade, $50 \%$ dos idosos do G1 apresentaram sensibilidade normal, $35 \%$ tiveram sensibilidade protetora diminuída e $10 \%$, perda total da sensibilidade protetora. Já no G2, 40\% apresentaram sensibilidade normal, 20\% tiveram sensibilidade protetora diminuída e $40 \%$ apresentaram perda total da sensibilidade protetora. O G2 registrou percentual de idosos com perda de sensibilidade protetora significativamente maior que o $\mathrm{G} 1$ $(\mathrm{p}=0,0028)$.

No TC6, o G1 apresentou melhor desempenho quando comparado ao G2. O G1 percorreu maior distância em metros, sendo esta diferença estatisticamente 
Tabela 1. Características demográficas e clínicas nos grupos com diabetes melitus e sem diabetes melitus

$\begin{array}{lccc}\text { Variáveis } & \text { G1 (n=20) } & \text { G2 (n=20) } & \text { Valor } p \\ \text { Idade (anos), média (DP) } & 70,6(5,68) & 68,1(5,67) & 30 \\ \text { Gênero feminino (\%) } & 15 & 70 & 0,4510 \\ \text { Gênero masculino (\%) } & 85 & 12,65(4,87) & 0,4510 \\ \text { Tempo de diagnóstico (anos), média (DP) } & 0 & 25,37(2,50) & 0,7300 \\ \text { IMC (kg/m²), média (DP) } & 25,12(2,22) & 0,93(0,10) & 90 \\ \text { ICQ (cm), média (DP) } & 0,87(0,59) & 10 & 35 \\ \text { Com hipertensão (\%) } & 75 & 35 \\ \text { Sem hipertensão (\%) } & 15 & 65 \\ \text { Com osteoporose (\%) } & 65 & 25 \\ \text { Sem osteoporose (\%) } & 35 & 75 \\ \text { Com artrose (\%) } & 40 & 15 \\ \text { Sem artrose (\%) } & 60 & 85 \\ \text { Com déficit visual (\%) } & 0 & 20 \\ \text { Sem déficit Visual (\%) } & 100 & 80 \\ \text { Com tabagismo (\%) } & 20 & 50 \\ \text { Sem tabagismo (\%) } & 80 & 50 \\ \text { Com etilismo (\%) } & 30 & 45 \\ \text { Sem etilismo (\%) } & 70 & 55 \\ \text { Com dislipidemia (\%) } & 20 & 80 & \end{array}$

G1: grupo com diabetes melitus; G2: grupo sem diabetes melitus; IMC: índice de massa corpórea; ICQ: índice cintura quadril; DP: desvio-padrão

Tabela 2. Variáveis no teste de caminhada de seis minutos nos grupos de idosos sem diabetes melitus e com diabetes melitus

$\begin{array}{lccc} & \text { G1 (n=20) } & \text { G2 (n=20) } & \text { Valor } p \\ \text { Distância percorrida (m), média (DP) } & 481,5(73,1) & 396,0(55,6) & 0,0003 \\ \text { Escala de Borg } & 4 \text { ou 6 } & 5 \text { ou 7 } & 0,0360\end{array}$

G1: grupo sem diabetes melitus; G2: grupo com diabetes melitus; DP: desvio-padrão

significativa, porém sem diferença significativa em relação à percepção subjetiva do esforço avaliada pela escala de Borg (Tabela 2).

Considerando o TDMF, não houve diferença estatisticamente significativa entre as atividades realizadas entre os idosos G1 e G2 (Tabela 3), assim como também não houve diferença em relação à pontuação máxima (escore total), atingida pelos dois grupos.

\section{DISCUSSÃO}

O DM está associado à incapacidade física e baixa qualidade de vida autorrelatada ${ }^{4,13}$. As incapacidades funcionais associadas ao diabetes podem levar a uma diminuição de mobilidade articular, da função muscular e funcionalidade de membros inferiores, podendo ser erroneamente atribuídas ao processo natural de envelhecimento $^{14}$

Um dos principais resultados do presente estudo transversal é que os idosos com DM apresentaram pior desempenho no TC6 quando comparados com idosos sem DM, pareados pelo sexo, idade e IMC, apontando para menor aptidão física dos G2 quando comparados aos $\mathrm{G}^{15,16}$. O TC6 possui boa correlação com o $\mathrm{VO}_{2}$ (consumo de oxigênio máximo). Grande parte de nossas atividades da vida diária é realizada em níveis submáximos, sendo assim, o TC6 reflete bem a capacidade funcional para as atividades do dia a dia ${ }^{17,18}$. Além disso, é um teste fácil de ser aplicado e bem tolerado pelos pacientes ${ }^{15,16}$.

Pessoas saudáveis caminham, durante o TC6, distâncias que variam entre 400 e $700 \mathrm{~m}^{19}$. No presente estudo foi observado que o grupo de DM percorreu distância menor durante o teste, alcançando média de 396 m, e o grupo de idosos sem a doença apresentou média dentro do previsto $-481,5 \mathrm{~m}$.

À avaliação da sensibilidade tátil no presente estudo, houve maior perda da sensibilidade tátil dos pés nos idosos com doença. Aproximadamente 50\% dos pacientes diabéticos com idade acima de 60 anos evidenciam neuropatia ${ }^{20}$.

Menz et al. ${ }^{21}$ avaliaram sujeitos do sexo masculino e feminino, com DM tipo 2 e neuropatia periférica, e idade entre 55 e 91 anos. Eles observaram que a velocidade de caminhada e distância do passo estavam associadas com o sentido de vibração e a sensibilidade tátil. A falta da sensibilidade é um dos sinais clínicos 
Tabela 3. Atividades do teste de desempenho físico modificado nos grupos de idosos sem diabetes melitus e com diabetes melitus

\begin{tabular}{|c|c|c|c|c|}
\hline & \multicolumn{2}{|c|}{ G1 } & \multicolumn{2}{|c|}{ G2 } \\
\hline & $\begin{array}{l}\text { Tempo gasto na } \\
\text { realização da tarefa (seg) }\end{array}$ & $\%$ & $\begin{array}{l}\text { Tempo gasto na } \\
\text { realização da tarefa (seg) }\end{array}$ & $\%$ \\
\hline \multirow[t]{4}{*}{ Pegar um livro } & 4,5 a 6,0 & 20 & 2,5 a 4,0 & 70 \\
\hline & 2,5 a 4,0 & 40 & $<2,0$ & 30 \\
\hline & $<2,0$ & 40 & & \\
\hline & $>20,0$ & 5 & & \\
\hline \multirow[t]{2}{*}{ Vestir e despir um casaco } & 10,5 a 15,0 & 90 & $>20,0$ & 5 \\
\hline & $<10,0$ & 5 & 10,5 a 15,0 & 95 \\
\hline \multirow[t]{2}{*}{ Pegar uma moeda no chão } & 2,5 a 4,0 & 95 & 2,5 a 4,0 & 100 \\
\hline & $>6,0$ & 5 & & \\
\hline \multirow[t]{3}{*}{ Levantar-se da cadeira } & $>11,8$ & 75 & $>11,8$ & 65 \\
\hline & 9,2 a 11,1 & 20 & 9,2 a 11,1 & 15 \\
\hline & 8,1 a 9,2 & 5 & 8,1 a 9,2 & 20 \\
\hline \multirow[t]{2}{*}{ Caminhar 15 m } & $<15,0$ & 95 & $<15,0$ & 100 \\
\hline & 15,5 a 20,0 & 5 & & \\
\hline \multirow{3}{*}{ Subir um lance de escada } & $>15,0$ & 35 & $>15,0$ & 10 \\
\hline & 10,5 a 15,0 & 40 & 10,5 a 15,0 & 60 \\
\hline & 5,5 a 10,0 & 25 & 5,5 a 10,0 & 30 \\
\hline Subir quatro lances de escadas & 4 lances & 100 & 4 lances & 100 \\
\hline \multirow[t]{4}{*}{ Girar $360^{\circ}$} & passos descontínuos & 10 & passos descontínuos & 5 \\
\hline & firmes, constante & 90 & firmes, constante & 95 \\
\hline & 4 & 5 & & \\
\hline & 5 & 5 & 5 & 5 \\
\hline Romberg progressivo & 6 & 90 & 6 & 95 \\
\hline
\end{tabular}

G1: grupo sem diabetes melitus; G2: grupo com diabetes melitus

mais encontrados em indivíduos com $\mathrm{DM}^{22}$. Essa perda da sensibilidade contribui para a diminuição das aferências do sistema de controle motor e, portanto, para a diminuição do equilíbrio. Tais comprometimentos geram alterações na marcha, postura, menor cadência, passos mais curtos e menor aceleração, assim como lentidão na correção de erros motores ou quando é necessário transpor obstáculos, favorecendo a ocorrência de quedas ${ }^{23}$.

Yavuzer et al. ${ }^{14}$ compararam diabéticos neuropáticos, não neuropáticos e grupo controle. Esses autores não observaram diferença significativa da velocidade da marcha entre os diabéticos neuropáticos e não neuropáticos. No entanto, foi encontrada diferença significativa entre os diabéticos e o grupo controle, o que mostra que a neuropatia periférica pode não ser a única razão para alterações na mobilidade e marcha dos idosos com DM.

Outro teste utilizado para avaliar a capacidade funcional foi o TDMF. É um instrumento sensível para predizer incapacidade futura, o que permite aos profissionais da saúde/reabilitação detectar precocemente as alterações funcionais do indivíduo por meio de escores comparativos. Quanto maior o escore total obtido pelo TDMF, melhor a performace durante as atividades funcionais ${ }^{10}$.
A maioria dos idosos do grupo de não DM (80\%) obteve escore de 28 ou 29 e $55 \%$ do grupo de DM, escore de 28 ou 29, não havendo diferença estatisticamente significativa. Da mesma forma, não houve diferença significativa entre os grupos em relação ao tempo gasto para realizar as atividades propostas pelo teste. A ausência de diferença entre grupos pode estar relacionada ao número restrito de sujeitos avaliados no presente estudo.

Estudos indicam que o TDMF detectou que doenças crônicas somáticas, tais como parkinsonismo, doença cardíaca, alteração visual e câncer, levam a um prejuízo no desempenho físico, mas não influenciam na incapacidade autorrelatada ${ }^{24-26}$. A presença dessas doenças crônicas pode conduzir à adoção de estratégias compensatórias para execução de tarefa, aumentando o tempo gasto para realizar atividades de vida diária. Seria plausível que o DM também fosse um fator a influenciar no desempenho dos participantes no TDMF. Entretanto, essa influência não foi detectada para o diabetes ${ }^{26}$ e nem comprovada pelo resultado do presente estudo.

O TDMF pode não ser sensível para detectar alterações funcionais prévias e sutis diferenças no tempo gasto para realizar atividades de vida diária em idosos com DM. No entanto, é necessário que novas pesquisas, 
com amostras maiores e maior controle sobre o DM em relação ao tipo e a presença de neuropatias e outras comorbidades, sejam conduzidas.

Ainda é necessário avançar no conhecimento das possíveis causas das incapacidades funcionais encontradas em idosos com DM. Analisar os fatores que levam o diabético a uma pior aptidão física é de fundamental importância clinica. O teste de sensibilidade dos pés, assim como o TC6, é de grande valia para o diagnóstico funcional e deve ser incluído na rotina das avaliações fisioterápicas desses pacientes, sendo de grande valia na prática clínica da fisioterapia na velhice, fase na qual se busca principalmente preservar a capacidade funcional dos idosos.

\section{CONCLUSÃO}

Os idosos DM apresentaram menor capacidade funcional, detectada pelo TC6 quando comparados aos idosos sem DM. Essa menor capacidade funcional pode estar associada à presença da doença e ou ser consequente ao fato do grupo apresentar menor sensibilidade protetora. $\mathrm{O}$ mesmo não pode ser observado no TDMF, talvez pelo fato do teste não ser sensível para detectar os possíveis comprometimentos dessa condição de saúde ou a amostra não ser suficiente para avaliar tais diferenças.

\section{REFERÊNCIAS}

1. Guimarães LHCT, Galdino DCA, Martins FLM, Vitorino DFM, Pereira KL, Carvalho EM. Comparação da propensão de quedas entre idosos que praticam atividade física e idosos sedentários. Rev Neurocienc. 2004;12(2):68-72.

2. Gregg EW, Beckles GL, Williamson DF, Leveille SG, Langlois JA, Engelgau MM , et al. Diabetes and physical disability among older U.S adults. Diabetes Care. 2000;23(9):1272-7.

3. Volpato S, Blaum C, Resnick H, Ferrucci L, Fried LP, Guralnik JM. Comorbidities and impairments explaining the association between diabetes and lower extremity disability: the women's health and aging study. Diabetes Care. 2002;25(4):678-83.

4. Araki $\mathrm{A}$, Ito $\mathrm{H}$. Diabetes mellitus and geriatric syndromes. Geriatr Gerontol Int. 2009;9(2):105-14.

5. Corriveau H, Hébert R, Raîche M, Dubois MF, Prince F. Evaluation of postural stability in elderly with stroke. Arch Phys Med Rehabil. 2004;85(7):1095-101.

6. Santos AA, Bertato FT, Montebelo MIL, Guirro ECO. Efeito do treinamento proprioceptive em mulheres diabéticas. Rev Bras Fisioter. 2008:12(3):183-7.
7. Chen HC, Ashton-Miller JA, Alexander NB, Schultz AB. Age effects on strategies used to avoid obstacles. Gait Posture. 1994;2:139-46.

8. Hanke TA, Rogers MW. Reliability of ground reaction force measurements during dynamic transitions from bipedal to singlelimb stance in healthy adults. Phys Ther. 1992;72(11):810-6.

9. Bertolucci PHF, Brucki SMD, Campacci S, Juliano Y. O mini-exame do estado mental em uma população geral. Impacto da escolaridade. Arq Neuropsiquiatr. 1998;56(3):605-12.

10. Mitre NCD, Dias RC, Dias JMD, Faria APS, Costa DC, Carvalho GM, et al. Adaptação para o português e confiabilidade de uma versão modificada do Physical Performance Test. Geriatria \& Gerontologia. 2008:3(2):104-109.

11. Cullen DL., Rodak B. Clinical utility of measures of breathlessness. Respir Care. 2002:47(9):986-93.

12. Souza A, Nery CAS, Marciano LHSC, Garbino JA. Avaliação da neuropatia periférica: correlação entre a sensibilidade cutânea dos pés, achados clínicos e eletroneuromiográficos. Acta Fisiatr. 2005;12(3):87-93.

13. Arkkila PE, Gautier JF. Musculoskeletal disorders in diabetes mellitus: an update. Best Pract Res Clin Rheumatol. 2003;17(6):945-70.

14. Yavuzer G, Yetkin I, Toruner FB, Kocan N, Bolukbasi N. Gait deviations of patients with diabetes mellitus: looking beyond peripheral neuropathy. Eura Medicophys. 2006;42(2):127-33.

15. Soares CPS, Pires SR, Britto RR, Parreira VF. Avaliação da aplicabilidade da equação de referência para estimativa de desempenho no teste de caminhada de 6 minutos em indivíduos saudáveis brasileiros. Rev Soc Cardiol Estado de São Paulo. 2004:14(1):1-8.

16. Enright PL. The six-minute walk test. Respir Care. 2003:48(8):783-5.

17. Hamilton DM, Haennel RG. Validity and reliability of the 6-minute walk test in a cardiac rehabilitation population. J Cardiopulm Rehabil. 2000;20(3):156-64.

18. Solway S, Brooks D, Lacasse Y, Thomas S. A qualitative systematic overview of the measurement properties of functional walk tests used in the cardiorespiratory domain. Chest. 2001;19(1):256-70.

19. Enright PL, Sherrill DL. Reference equations for six-minute in healthy adults. Am J Respir Crit Care Med. 1998;158(5 Pt 1):1384-7.

20. Pham H, Armstrong DG, Harvey C, Harkless LB, Giurini JM, Veves A. Screening techniques to identify people at high risk for diabetic foot ulceration: a prospective multicenter trial. Diabetes Care. 2000;23(5):606-11.

21. Menz HB, Lord SR, St George R, Fitzpatrick RC. Walking stability and sensoriomotor function in older people with diabetic peripheral neuropathy. Arch Phys Med Rehabil. 2004;85(2):245-52.

22. Corriveau H, Prince F, Hébert R, Raîche M, Tessier D, Maheux P, et al. Evaluation of postural stability in elderly with diabetic neuropathy. Diabetes Care. 2000;23(8):1187-91.

23. Cavanagh PR, Hewitt Jr FG, Perry JE. In-shoe plantar pressure measurement: a review. The Foot. 1992;2(4):185-94.

24. Fried LP, Herdman SJ, Kuhn KE, Rubin G, Turano K. Preclinical disability: hypotheses about the bottom of the iceberg. J Aging Health. 1991;3:285-300.

25. Leibovici D, Curtis S, Ritchie K. The application of disability data from epidemiological surveys to the development of indicators of service needs for dependent elderly people. Age Ageing. 1995;24(1):12-20.

26. Rozzini R, Frisoni GB, Ferrucci L, Barbisoni P, Bertozzi B, Trabucchi $M$. The effect of chronic diseases on physical function. Comparison between activities of daily living scales and physical performance test. Age Ageing. 1997;26(4):281-7. 